\title{
A spatial and temporal analysis of paediatric central nervous system infections from 2005 to 2015 in Ho Chi Minh City, Vietnam
}

\author{
N. T. HO ${ }^{1}$, V. M. T. HOANG ${ }^{2}$, N. N. T. LE ${ }^{3}$, D. T. NGUYEN ${ }^{4}, A \cdot$ TRAN $^{5}$, \\ D. KAKI ${ }^{6}$, P. M. TRAN ${ }^{7}$, C. N. THOMPSON ${ }^{7}$, M. N. Q. NGO ${ }^{3}$, K. H. TRUONG ${ }^{3}$, \\ H. T. NGUYEN ${ }^{3}$, T. M. HA ${ }^{2}$, C. V. V. NGUYEN ${ }^{4}$, G. E. THWAITES ${ }^{7,8}$, \\ K. T. THAKUR ${ }^{1}$, D. HESDORFFER ${ }^{1}$ AND S. BAKER ${ }^{7,8,9} *$ \\ ${ }^{1}$ Columbia University Medical Center, New York City, New York, USA \\ ${ }^{2}$ Children's Hospital 2, Ho Chi Minh City, Vietnam \\ ${ }^{3}$ Children's Hospital 1, Ho Chi Minh City, Vietnam \\ ${ }^{4}$ Hospital for Tropical Diseases, Ho Chi Minh City, Vietnam \\ ${ }^{5}$ Saint Louis University, Saint Louis, Missouri, USA \\ ${ }^{6}$ Princeton University, Princeton, New Jersey, USA \\ ${ }^{7}$ Oxford University Clinical Research Unit, Wellcome Trust Major Overseas Programme, \\ Ho Chi Minh City, Vietnam \\ ${ }^{8}$ Centre for Tropical Medicine and Global Health, Nuffield Department of Medicine, \\ University of Oxford, Oxford, UK \\ ${ }^{9}$ The Department of Medicine, University of Cambridge, Cambridge, UK
}

Received 1 February 2017; Final revision 19 July 2017; Accepted 7 September 2017; first published online 24 October 2017

\section{SUMMARY}

Central nervous system infections (CNSI) are a leading cause of death and long-term disability in children. Using ICD-10 data from 2005 to 2015 from three central hospitals in Ho Chi Minh City (HCMC), Vietnam, we exploited generalized additive mixed models (GAMM) to examine the spatial-temporal distribution and spatial and climatic risk factors of paediatric CNSI, excluding tuberculous meningitis, in this setting. From 2005 to 2015, there were 9469 cases of paediatric CNSI; $33 \%$ were $\leqslant 1$ year old at admission and were mainly diagnosed with presumed bacterial CNSI (BI) $(79 \%)$, the remainder were $>1$ year old and mainly diagnosed with presumed non-bacterial CNSI (non-BI) (59\%). The urban districts of HCMC in proximity to the hospitals as well as some outer districts had the highest incidences of BI and non-BI; BI incidence was higher in the dry season. Monthly BI incidence exhibited a significant decreasing trend over the study. Both BI and non-BI were significantly associated with lags in monthly average temperature, rainfall, and river water level. Our findings add new insights into this important group of infections in Vietnam, and highlight where resources for the prevention and control of paediatric CNSI should be allocated.

Key words: Encephalitis, epidemiology, spatial temporal, paediatric, meningitis.

\footnotetext{
* Author for correspondence: Professor S Baker, Oxford University Clinical Research Unit, 764 Vo Van Kiet, Quan 5, Ho Chi Minh City, Vietnam.

(Email: sbaker@oucru.org)
}

\section{INTRODUCTION}

Infections of the central nervous system (CNSI) are among the leading causes of death and severe longterm disability in children in developing countries [1-3]. The clinical presentations, treatment regimens, 
and outcomes of CNSI are heterogeneous and highly dependent on a multitude of variables including the neurovirulence of the pathogen, host susceptibility, epidemiological factors and variation in environmental exposures. The etiological agents (including bacteria and viruses) and the modes of transmission (such as vector-borne or person-to-person) of CNSI are additionally diverse, and in many cases unidentified [1, 2].

In a low-resource country like Vietnam, the specific pathogen associated with an individual CNSI is often undetermined. Studies designed to identify the etiological agents of CNSI in Vietnam fail to identify a pathogen in approximately half of the CNSI patients. Among those identified, the most common bacterial pathogens of paediatric CNSI in Vietnam are Streptococcus pneumoniae and Haemophilus influenzae type $\mathrm{b}$ (Hib), whilst the most common viral pathogens are Japanese encephalitis (JE), Dengue virus, and a range of enteroviruses [4-6]. Although a vaccine against JE virus was introduced in the Expanded Programme on Immunization (EPI) in Vietnam in 2006, mosquito-borne viral encephalitis is still considered to be a major public health concern in Vietnam [7]. Vaccines against $S$. pneumoniae and Neisseria meningitidis (meningococcus) are available in Vietnam but are expensive and not yet incorporated into EPI schedule. With support from the Global Alliance for Vaccine and Immunization (GAVI), Vietnam introduced a Hib vaccine in 2010, which was incorporated into a pentavalent formulation (diphtheria, tetanus, pertussis, Hepatitis B, and Hib). The World Health Organization (WHO) estimated that the national coverage of $\mathrm{Hib}$ vaccine in Vietnam increased from $63 \%$ in 2010 to $97 \%$ in 2015 [8]. However, GAVI will not continue their funding for this initiative after 2016 and as the results, the Vietnamese government will face a sixfold increase in the cost of Hib vaccines [9].

There is evidence to suggest that various spatial, climatic and socio-demographic risk factors may be associated with the incidence of CNSI in low-middle income countries such as those located in the African sub-Saharan meningitis belt and Asia (e.g. vector-born encephalitis is associated with proximity to rice cultivation and pig rearing and bacterial meningitis is associated with seasonality (the winter months) and high population densities) [10-17]. Vietnam faces continuing lack of access to costly vaccines against bacterial and viral etiologies of CNSI. Therefore, we aimed to provide a better understanding of sub-populations at risk of CNSI so they can be targeted for improved immunization strategies. We conducted an analysis of the spatial distribution and climatic risk factors of paediatric CNSI in Ho Chi Minh City (HCMC), the major economic centre in the south of Vietnam, which may assist in efficiently targeting prevention and control methods for CNSI, such as vector control measures and immunization programmes.

\section{METHODS}

\section{Data sources}

Data for this study were derived from the electronic hospital databases of all paediatric (aged $<16$ years) Vietnamese inpatients admitted to three large main hospitals located in central HCMC: Children's Hospital one (CH1), Children's Hospital two (CH2), and the Hospital for Tropical Diseases (HTD). These hospitals receive most of the paediatric patients especially those requiring hospital care from HCMC, an economic centre with around 8 million population in the South of Vietnam. Data from the two paediatric hospitals (CH1 and CH2) were obtained between 2005 and 2015, while the data from HTD were obtained between 2008 and 2015. All the three study hospitals employed the International Classification of Disease version 10 (ICD10) for coding disease diagnoses. Some routine electronic hospital data were available from these hospitals before the study period; however, we only utilized data generated in years that used ICD10 coding to ensure the consistency in database quality. We only included patients residing in HCMC, excluding patients from surrounding provinces, in this analysis anticipating the data were representative of HCMC population.

We classified CNSI into two major groups based on the ICD10 codes outlined by the WHO: (1) Presumed bacterial CNSI (BI), which were amalgamated under the ICD10 codes G00-G01 and G03 for bacterial meningitis, not elsewhere classified, meningitis in bacterial diseases classified elsewhere and for meningitis due to other and unspecified causes respectively. (2) Presumed non-bacterial CNSI (non-BI), which incorporated the ICD10 codes A80-A89 for viral and prion infections of the central nervous system, G02 for meningitis in other infectious and parasitic diseases classified elsewhere, G04-G05 for encephalitis, myelitis and encephalomyelitis (online Supplementary Table S1). As most of the paediatric patients with tuberculous meningitis (TBM) are treated at another hospital 
specialized for tuberculous diseases in HCMC, ICD10 codes for TBM (A17.0) was not included in our analysis. Data from patients with the selected ICD10 codes who resided in HCMC were extracted and analyzed. Each patient record included age, sex, date of admission, date of discharge, ICD10 code at discharge, and residential district.

HCMC is subdivided into 24 districts (land areas ranging from 4.18 to $704 \cdot 22 \mathrm{~km}^{2}$ ). There are five rural districts (population density ranging from 100 to 3326 people $/ \mathrm{km}^{2}$ ) and 19 urban districts (population density ranging from 2360 to 45582 people $/ \mathrm{km}^{2}$ ) [18]. There are two distinct seasons in HCMC: a dry season (from December to April) and a rainy season (from May to November). Various characteristics of the districts, including population density $\left(1000 / \mathrm{km}^{2}\right)$, average number of pig herds with pigs aged $>2$ months, land usage for rice cultivation, and population data were obtained from the HCMC statistical office [18]. As disaggregated population figures for those aged $<16$ years per district were unavailable, the total population was used to estimate disease incidence. Patient addresses in HCMC were geocoded to district centroids. City level monthly average climate data during 2005-2015 were obtained from the Ministry of Natural Resources and Environment of Vietnam. These data included monthly average temperature $\left(\right.$ median $=28 \cdot 3^{\circ} \mathrm{C}$, range $\left.=25 \cdot 9-31 \cdot 3^{\circ} \mathrm{C}\right)$, relative humidity $($ median $=74 \cdot 4 \%$, range $=60 \cdot 4 \%$ to $83 \cdot 2 \%$ ), rainfall $($ median $=4.9 \mathrm{~mm}$, range $=0-17 \mathrm{~mm}$ ), and water level of the Don Dien River (median $=10.6 \mathrm{~cm}$, range $=-19 \cdot 3$ to $39 \cdot 1 \mathrm{~cm}$ ) [19]. District elevation data were obtained from the CGIAR Consortium for Spatial Information (CGIAR-CSI) [20].

\section{Spatial mapping}

To reduce the variation of raw incidence estimates due to the variation in population sizes, district incidence rates (number of CNSI cases per 100000 district total population for the entire study period) were smoothed using local empirical Bayes estimates for rates reduced to a neighborhood mean. The neighborhoods were identified by a neighborhood list based on districts with contiguous boundaries ( $\mathrm{R}$ package spdep version 0.5-88) [21]. Kulldorff and Nagarwalla's method over district centroids was used to detect clusters of disease [22].

A Poisson generalized linear model was used to explore the relationship between district CNSI incidence and district characteristics (the logarithm of the distance from district centroids to the nearest study hospital, district population density, number of pigs in herds with pigs aged $>2$ months by district, $\%$ area of district used for rice cultivation, and district elevation). Confidence intervals (CI) and statistical tests were based on robust standard errors to control for mild violations of the distributional assumption that the variance equals the mean [23].

\section{Time series analysis}

The time series of citywide monthly incidence of CNSI (number of monthly cases per 100000 total population of that month) were examined for seasonality, time trends, and associations with climatic covariates by Poisson generalized additive mixed models (GAMM) implemented in the $\mathrm{R}$ package mgev version 1.8-7 [24, 25]. Seasonal cycles and time trends of monthly CNSI incidence were evaluated by the basic GAMM models comprising the month of year $(\mathrm{Ms}=1-12)$ as cyclic cubic regression splines and the month of the whole study period $(\mathrm{Mt}=1-96)$ as cubic regression spline. As monthly CNSI incidence may be associated with the CNSI incidence and climatic conditions of the previous months, in addition to the elements in the basic model, the full GAMM model also contained lags of CNSI monthly incidence of up to 4 months, concurrent climatic condition and lags of climatic condition up to 4 months. Adjusted $R^{2}$ and model diagnostic plots were used to evaluate the performance of the GAMM models. Temporal correlation of the model residuals was evaluated by autocorrelation function (ACF) and partial ACF.

Notably, the CNSI data prior to 2008 may contain ICD10 codes for neurological complications associated with hand, foot, and mouth diseases (HFMD) (potentially coded as A85.0 for enteroviral encephalitis). From 2008, patients with HFMD were coded with separate ICD10 codes (B08.4). HFMD is currently epidemic in Vietnam and may have different spatiotemporal patterns and climatic risk factors in comparison with other CNSI. Therefore, to avoid the bias induced by the inclusion of HFMD in these data, only CNSI data from 2008 to 2015 were used for the analysis of temporal patterns and climatic risk factors. Spatial mapping and statistical analyses were stratified by BI and non-BI and all subsequent analyses were performed in $\mathrm{R}$ version $3 \cdot 2 \cdot 2$ [26].

\section{RESULTS}

\section{Features of paediatric CNSI in HCMC}

From 2005 to 2015 , there were 9469 paediatric $(<16$ years old) patients residing in HCMC admitted to 
the three study hospitals for CNSI $(1 \cdot 5 \%$ of all paediatric admissions). There were 5182 patients ( $55 \%$ of all CNSI admissions) with ICD10 codes synonymous with presumed BI and 4287 patients $(45 \%)$ with ICD10 codes synonymous with presumed non-BI. The BI patients were significantly younger than the non-BI patients (median (interquartile-IQR) $=1 \cdot 1$ years $(0 \cdot 1,5 \cdot 0$ years $)$ vs. $3 \cdot 8$ years $(1 \cdot 6,6 \cdot 9$ years $)$, $P<0 \cdot 0001)$. CNSI patients aged $\leqslant 1$ year at admission accounted for 33\% (3110/9469) and the vast majority $(2469 / 3110 ; 79 \%)$ were diagnosed as having presumed BI. Whereas, the majority of CNSI patients aged $>1$ year were diagnosed as presumed non-BI (3597/5872; 59\%). BI patients were hospitalized for a significantly longer period of time than non-BI patients $($ median $(\mathrm{IQR})=13 \cdot 0$ days $(8 \cdot 0,20 \cdot 0$ days $)$ vs. $4 \cdot 0$ days (3.0, $7 \cdot 0$ days), $P<0 \cdot 0001)$. Mortality of BI patients was significantly lower than the non-BI patients $(1 \%$ vs. $4 \%, P<0.0001)$ (Tables 1 and 2$)$.

Overall, the most commonly diagnosed groups were bacterial meningitis, not elsewhere classified (ICD10 code $=$ G00) $(3722 / 9469 ; 39 \%)$, meningitis due to other and unspecified causes (G03) (1460/9469; 15\%), viral meningitis (A87) (1241/9469; 13\%), meningitis in other infectious and parasitic diseases classified elsewhere (G02) (1118/9469; 12\%), other viral encephalitis, not elsewhere classified (A85) (1071/9469; 11\%) and encephalitis, myelitis, and encephalomyelitis (G04) (657/9469; $7 \%$ ) (Table 2). A more detailed outline of the diagnoses, ICD10 codes and explanation for categorizing ICD10 codes from our data into BI and non-BI groups are shown in online Supplementary Table S1.

\section{The spatial distribution of paediatric CNSI in HCMC}

Over the entire study period (2005-2015), the median district incidence of BI (number of BI cases per 100 000 district population) was $64 \cdot 5$ (range $=40 \cdot 3$, $105 \cdot 1$ ); the median district incidence of non-BI was $51 \cdot 4$ per $100000($ range $=32 \cdot 1,84 \cdot 2)$. The highest incidences of BI and non-BI were identified in the urban districts of HCMC that were in the closest proximity to $\mathrm{CH} 1$ and $\mathrm{CH} 2$ (districts 3, 10, 11, 5, 6, Tan Binh, and Tan Phu) and some rural districts located significant distances from the study hospitals (Hoc Mon and Binh Chanh) $(P<0 \cdot 0001)$ (Fig. 1). There were two periods in which there were notable peaks in non-BI and BI, these occurred in 2007 and 2009, respectively. The increase in non-BI and BI incidence during these periods occurred concurrently in the same nine districts identified above (online supplementary Figs S1,
S2, S3 and S4). We also observed an overall decrease in BI incidence in these nine districts between 2013 and 2015 (online supplementary Fig. S1).

The district level incidence of BI over the study period was significantly associated with the logarithm of Euclidean distance from district centroids to the nearest study hospital (rate ratio (RR) for two times increase in distance $=0.915,95 \%$ confidence interval (CI) $0 \cdot 875-0.957)$ and district population density (RR for 1 unit $\left(1000 / \mathrm{km}^{2}\right)$ increase in population density $=1 \cdot 006,95 \%$ CI 1.001-1·011). However, in a multivariate model containing both the logarithm of distance to hospital and population density, only the logarithm of distance to hospital remained statistically significant $(\mathrm{RR}=0.911,95 \%$ CI $0.850-0.977)$. The district incidence of non-BI was not significantly associated with any of the district variables examined (online supplementary Fig. S5).

\section{Temporal patterns and climatic risk factors of paediatric CNSI}

The monthly incidence of BI (number of monthly cases per 100000 population) ranged from $0 \cdot 19$ to 1.61 (median $=0 \cdot 48)$ and the monthly incidence of non-BI ranged from 0.07 to 1.28 (median $=0 \cdot 29)$ per 100000 . There was one major peak of BI, this occurred in the dry season of 2009 and was associated with the ICD10 code of bacterial meningitis not elsewhere classified (G00) $(68 \%$ of BI patients $(41 \%$ of all CNSI patients) during that period). Generally, most periods with a high incidence of BI were associated with the dry season and were preceded by a smaller peak in the late months of rainy season. There was a single exceptionally large peak of non-BI in the wet season of 2007; this was related to enteroviral encephalitis (A85.0) $(88 \%$ of non-BI patients $(70 \%$ of all CNSI patients) during that period). After this potential outbreak, the incidence of non-BI exhibited sporadic peaks in 2009 and 2010, which were mainly associated with the ICD10 code of meningitis in viral diseases classified elsewhere (G02.0) $(74 \%$ and $47 \%$ of non-BI patients during those periods, respectively). Again, a peak in non-BI in 2012 and 2015 was generally associated with viral meningitis (A87) (59\% and $63 \%$, respectively). With the exception of the large peak in non-BI during the rainy season of 2007, the highest incidences of non-BI displayed a similar pattern of peaking mostly in the dry season. Overall, the climatic data displayed exhibited similar seasonal patterns over the 11-year study period (Fig. 2). 
Table 1. The characteristics of paediatric patients with presumed bacterial and non-bacterial central nervous system infections (CNSI) in Ho Chi Minh from 2005 to 2015

\begin{tabular}{|c|c|c|c|c|}
\hline Patient characteristic & $\begin{array}{l}\text { All CNSI patients } \\
(N=9469)\end{array}$ & $\begin{array}{l}\text { Presumed bacterial } \\
\text { CNSI } \\
(N=5182)\end{array}$ & $\begin{array}{l}\text { Presumed non-bacterial } \\
\text { CNSI } \\
(N=4287)\end{array}$ & $P$ value \\
\hline $\begin{array}{l}\text { Age } \\
(\text { median(interquartile) }) \\
(n=9215)\end{array}$ & $2 \cdot 80(0 \cdot 40,6 \cdot 00)$ & $1 \cdot 10(0 \cdot 10,5 \cdot 00)$ & $3 \cdot 80(1 \cdot 60,6 \cdot 90)$ & $<0.0001^{*}$ \\
\hline $\begin{array}{l}\text { Days in hospital } \\
\text { (median(interquartile)) } \\
(n=9469)\end{array}$ & $8 \cdot 00(4 \cdot 00,15 \cdot 00)$ & $13 \cdot 00(8 \cdot 00,20 \cdot 00)$ & $4 \cdot 00(3 \cdot 00,7 \cdot 00)$ & $<0.0001^{*}$ \\
\hline Sex (male) & $6028 / 9469(64 \%)$ & $3252 / 5182(63 \%)$ & $2776 / 4287(65 \%)$ & $0 \cdot 046^{\dagger}$ \\
\hline Outcome & & & & $<0 \cdot 0001^{\dagger}$ \\
\hline Dead & $188 / 7923(2 \%)$ & $29 / 4228(1 \%)$ & $159 / 3695(4 \%)$ & \\
\hline Discharged & $7635 / 7923(96 \%)$ & $4148 / 4228(98 \%)$ & $3487 / 3695(94 \%)$ & \\
\hline Presumed Worse & $92 / 7923(1 \%)$ & $48 / 4228(1 \%)$ & $44 / 3695(1 \%)$ & \\
\hline Unknown & $8 / 7923(0 \%)$ & $3 / 4228(0 \%)$ & $5 / 3695(0 \%)$ & \\
\hline
\end{tabular}

$N$ : total number of patients in the group; $n$ : number of patients with available information.

* $P$-value from Wilcoxon's rank sum test comparing presumed bacterial infections $v s$. presumed non-bacterial infections.

$\dagger P$-value from Fisher's exact test comparing presumed bacterial infections $v s$. presumed non-bacterial infections.

A basic GAMM model indicated that BI exhibited significant seasonality $(P<0 \cdot 0001)$. Overall, the monthly incidence of BI significantly decreased over time (incidence rate ratio (RR) for 1 month increase in time $=0 \cdot 61,95 \%$ CI $0 \cdot 38-0 \cdot 98, P=0 \cdot 0441)$ and the monthly incidence of non-BI significantly increased over time $(\mathrm{RR}=2 \cdot 04,95 \%$ CI $1 \cdot 19-3 \cdot 5$, $P=0 \cdot 0113)$ (Table 3).

The monthly incidence of BI was significantly associated with BI incidence 1 and 4 month previously (i.e. 1 and 4 month lags of monthly BI incidence) $(\mathrm{RR}=1.73,95 \%$ CI $1.22-2 \cdot 45, P=0.003$ and $\mathrm{RR}=$ $0 \cdot 55,95 \%$ CI $0 \cdot 39-0 \cdot 78, P=0.0015$, respectively). Additionally, the monthly incidence of BI was significantly associated with a 1 month lag in monthly average temperature $\left(\mathrm{RR}\right.$ for $1{ }^{\circ} \mathrm{C}$ increase in temperature $=1 \cdot 2$, $95 \%$ CI $1 \cdot 05-1 \cdot 37, \quad P=0.0084)$, average monthly rainfall $(\mathrm{RR}$ for $1 \mathrm{~mm}$ increase in rainfall $=1 \cdot 04,95 \%$ CI 1.01-1.06, $P=0 \cdot 0137$ ), and was concurrent (and 1 and 2 month lags) with the average monthly river water level (RR for $1 \mathrm{~cm}$ increase in river water level $=1 \cdot 01,95 \%$ CI $1 \cdot 0-1 \cdot 02, P=0.0266$, and RR $=0.99$, $95 \%$ CI $0.98-0 \cdot 99, P=0 \cdot 0364$ and $\mathrm{RR}=1 \cdot 02,95 \% \mathrm{CI}$ $1 \cdot 0-1 \cdot 03, P=0 \cdot 0169$, respectively) (Table 3 ).

The monthly incidence of non-BI was significantly associated with non-BI incidence 1 and 4 months previously (i.e. 1 and 4 month lags of monthly non-BI incidence) $(\mathrm{RR}=2 \cdot 83,95 \%$ CI $1 \cdot 77-4 \cdot 53, P=0.0001$ and $\mathrm{RR}=0 \cdot 56,95 \%$ CI $0 \cdot 35-0 \cdot 91, P=0 \cdot 0218$, respectively), 1 and 4 month lags in temperature $(\mathrm{RR}=1 \cdot 51$,
95\% CI $1 \cdot 24-1 \cdot 82, P=0 \cdot 0001$ and $\mathrm{RR}=1 \cdot 2,95 \% \mathrm{CI}$ $1 \cdot 04-1 \cdot 39, P=0 \cdot 0157$, respectively), 1 and 3 month lags in rainfall $(\mathrm{RR}=1 \cdot 05,95 \%$ CI $1 \cdot 02-1 \cdot 09, P=$ $0 \cdot 0058$ and $\mathrm{RR}=0 \cdot 96,95 \%$ CI $0 \cdot 93-0 \cdot 99, P=0 \cdot 0111$, respectively), and water level ( $\mathrm{RR}=1 \cdot 01,95 \% \mathrm{CI} 1 \cdot 0$ $1 \cdot 03, P=0.0459)$ (Table 3). The full GAMM models fitted the data well for both BI (adjusted $R^{2}=0 \cdot 77$ ) and non-BI (adjusted $R^{2}=0 \cdot 74$ ) (Fig. 3 and online supplementary Fig. S6). Lastly, a temporal correlation of monthly incidence was well controlled for both non-BI and BI in the full GAMM models (online supplementary Fig. S7).

\section{DISCUSSION}

Our study examined the spatial distribution, temporal patterns, and climatic risk factors for paediatric CNSI in southern Vietnam over an 11-year period. Our data show that, over the study period, presumed BI were the major contributor to CNSI in neonates and infants, while presumed non-BI were more common in older children. These data are consistent with the findings from other countries [27, 28]. The longer hospitalization periods associated with $\mathrm{BI}$, in comparison with non-BI patients, may be attributed to the longer intravenous treatment of bacterial CNSI in neonates and infants [29]. We additionally identified a higher mortality rate in the non-BI group. This maybe due to the fact that although some non-BI diseases may be self-limiting, there is currently no specific treatment 
Table 2. The diagnostic groups and ICD10 codes of paediatric patients with central nervous system infections (CNSI) by age at admission in Ho Chi Minh from 2005 to 2015

\begin{tabular}{|c|c|c|c|c|c|}
\hline Diagnosis (ICD10) & $\begin{array}{l}\text { All patients } \\
(N=9469)(\%)\end{array}$ & $\begin{array}{l}<1 \text { month } \\
(N=1647)(\%)\end{array}$ & $\begin{array}{l}1 \text { month-1 year } \\
(N=1463)(\%)\end{array}$ & $\begin{array}{l}1 \text { year }-5 \text { years } \\
(N=3258)(\%)\end{array}$ & $\begin{array}{l}>5 \text { years } \\
(N=2847)(\%)\end{array}$ \\
\hline Presumed bacterial CNSI* & $5182 / 9469(55)$ & $1575 / 1647(96)$ & $894 / 1463(61)$ & $1285 / 3258(39)$ & $1223 / 2847(43 \%)$ \\
\hline Bacterial meningitis, not elsewhere classified (G00) & $322 / 9469(39)$ & $1445 / 1647(88)$ & $634 / 1463(43)$ & $744 / 3258(23)$ & $696 / 2847(24 \%)$ \\
\hline Meningitis due to other and unspecified causes (G03) & $1460 / 9469(15)$ & $130 / 1647(8)$ & $260 / 1463(18)$ & $541 / 3258(17)$ & $527 / 2847(19 \%)$ \\
\hline Presumed non-bacterial CNSI* & $4287 / 9469(45)$ & $72 / 1647(4)$ & $569 / 1463(39)$ & $1973 / 3258(61)$ & $1624 / 2847(57 \%)$ \\
\hline Unspecified viral encephalitis (A86) & $137 / 9469(1)$ & $2 / 1647(0)$ & $18 / 1463(1)$ & $43 / 3258(1)$ & $74 / 2847(3 \%)$ \\
\hline Encephalitis, myelitis and encephalomyelitis $(\mathrm{G} 04) \dagger$ & $657 / 9469(7)$ & $9 / 1647(1)$ & $84 / 1463(6)$ & $252 / 3258(8)$ & $303 / 2847(11 \%)$ \\
\hline Encephalitis, myelitis and encephalomyelitis in diseases classified elsewhere (G05) & $16 / 9469(0)$ & $0 / 1647(0)$ & $0 / 1463(0)$ & $7 / 3258(0)$ & $7 / 2847(0 \%)$ \\
\hline Meningitis in other infectious and parasitic diseases classified elsewhere (G02) & $1118 / 9469(12)$ & $14 / 1647(1)$ & $96 / 1463(7)$ & $429 / 3258(13)$ & $567 / 2847(20 \%)$ \\
\hline Mosquito-borne viral encephalitis (A83) & $46 / 9469(0)$ & $0 / 1647(0)$ & $5 / 1463(0)$ & $12 / 3258(0)$ & $28 / 2847(1 \%)$ \\
\hline Other viral encephalitis, not elsewhere classified (A85) & $1071 / 9469(11)$ & $2 / 1647(0)$ & $264 / 1463(18)$ & $761 / 3258(23)$ & $38 / 2847(1 \%)$ \\
\hline Unspecified viral infection of central nervous system (A89) & $1 / 9469(0)$ & $0 / 1647(0)$ & $0 / 1463(0)$ & $0 / 3258(0)$ & $1 / 2847(0 \%)$ \\
\hline Viral meningitis (A87) & $1241 / 9469(13)$ & $45 / 1647(3)$ & $102 / 1463(7)$ & $469 / 3258(14)$ & $606 / 2847(21 \%)$ \\
\hline
\end{tabular}

ICD10, International Classification of Disease version 10.

* Fisher's exact test comparing the proportion of presumed bacterial CNSI and presumed non-bacterial CNSI across age groups $P$-value $<0 \cdot 0001$.

$\uparrow$ ICD10 code G04 may include both bacterial and viral CNSI. Most of the ICD codes in our databases only contained general ICD codes like G04 therefore it was not straightforward how to classify G04 codes into BI or non-BI group based on the data in our databases. However, we learned that, clinically, most of the CNSI cases coded with G04 in our three study hospitals were non-BI. Therefore, for the purpose of our analysis, all CNSI cases with ICD10 code G04 were grouped as non-BI.

More detailed diagnoses and ICD10 codes for the whole study period are in online supplementary Table S1. 


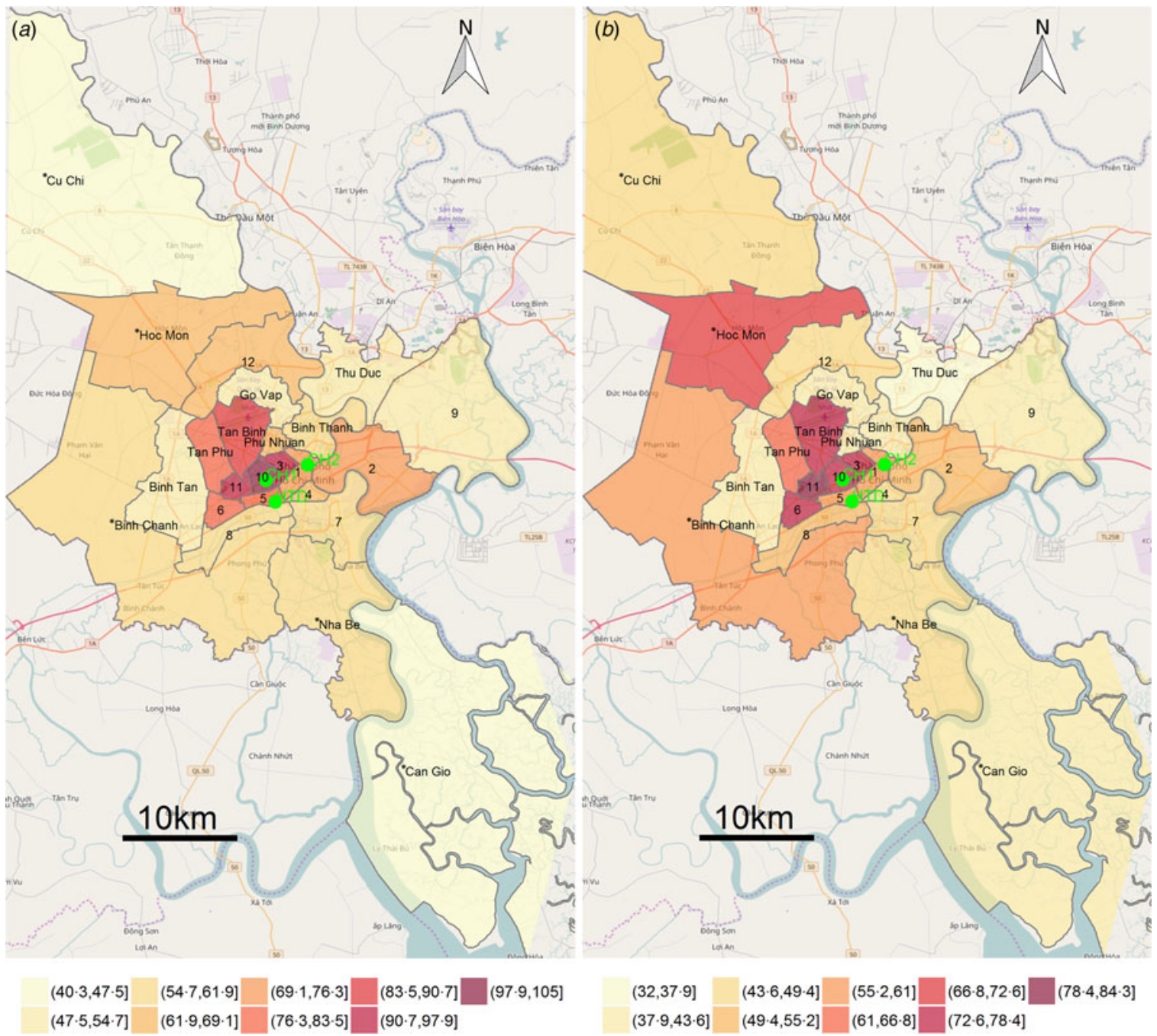

Fig. 1. The empirical Bayesian estimated incidence rates (EBR) of paediatric CNSI of presumed bacterial and non-bacterial origin in Ho Chi Minh City from 2005 to 2015. (a) District level EBR per 100000 district total population of presumed bacterial CNS infections for the whole study period. (b) District level EBR per 100000 district total population of presumed non-bacterial CNS infections for the whole study period. The green points show the locations of the study hospitals, $\mathrm{CH} 1$; Children hospital 1, CH2; Children hospital 2, and HTD; the Hospital for Tropical Diseases. An asterisk highlights rural districts.

for the majority of viral-associated CNSI, and many agents of viral CNSI, such as JE are fatal or can cause severe complications.

Given the hospital referral patterns and population structures of HCMC, it is unsurprising that the incidences of CNSI were higher in the districts in close proximity to the hospitals. However, a high incidence of CNSI, specifically during the major disease peaks, was also identified in the rural districts of Hoc Mon and Binh Chanh, which are located in the outer part of the city and some distance from the study hospitals. It is likely that a high incidence of CNSI in these districts may truly reflect higher incidences of CNSI in these districts in comparison with other HCMC districts, as the majority of paediatric patients with CNSI require hospital care.

The exceptional disease peak observed for enteroviral encephalitis $(\mathrm{ICD} 10=\mathrm{A} 85 \cdot 0)$ in 2007 probably includes patients with CNS complications associated with HFMD. There were no patients entered into the databases with the ICD10 A85.0 (HFMD) after 2008, because all patients with HFMD were coded with a different ICD10 code (B08.4) from 2008 onwards. Our time series analysis was conducted on data from 2008 to 2015; therefore our findings are not likely to be biased by the inclusion of patients with HFMD prior to 2008 . 


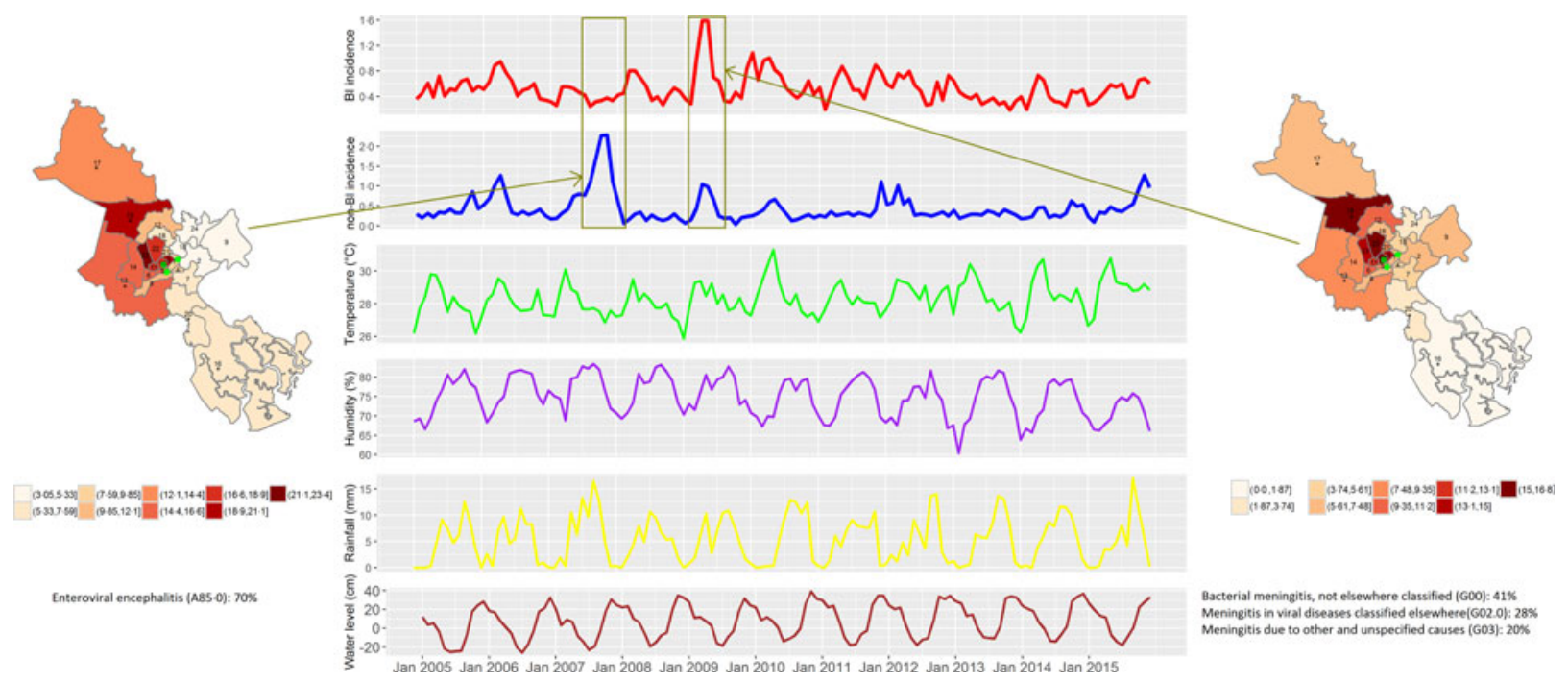

Fig. 2. The monthly incidence of paediatric presumed bacterial and non-bacterial CNSI vs. climate covariates and maps for the major outbreaks. Plots showing time series data for (from top to bottom); BI (presumed bacterial CNSI), non-BI (presumed non-bacterial $(\mathrm{CNSI})$ ), temperature $\left({ }^{\circ} \mathrm{C}\right)$, humidity $(\%)$, rainfall $(\mathrm{mm})$, and river water level $(\mathrm{cm})$. Adjacent maps show the district incidence (number of cases per 100000 district total population) of non-BI and BI during the two major outbreaks and most commonly recorded ICD10 codes at these time points. The green points show the locations of the study hospitals. The districts are labelled and an asterisk highlights rural districts. 1-12: district 1-12; 13-24: district Binh Chanh, Binh Tan, Binh Thanh, Can Gio, Cu Chi, Go Vap, Hoc Mon, Nha Be, Phu Nhuan, Tan Binh, Tan Phu, Thu Duc, respectively.

A high incidence of BI in the dry season (winter and spring months) is consistent with a high incidence in the dry season of bacterial meningitis in many countries $[11,12]$. The relatively strong association between BI and non-BI incidence with 1-month-lag of temperature may explain the stronger peaks of BI and non-BI mostly in the dry season. The slightly significant association of $\mathrm{BI}$ incidence with concurrent or lags of rainfall and river water level may explain the smaller peaks in BI incidence in the late months of rainy season. This may also reflect the heterogeneity of the BI and non-BI groups in our data in term of microbiological subclasses as well as corresponding temporal pattern and association with climatic risk factors. The significant association of both BI and non-BI incidence with concurrent or lags of temperature, rainfall and river water level suggests that climatic factors do play a critical role in the fluctuations of CNSI incidence. Climatic factors have also found to be associated with seasonality and temporal patterns of bacterial meningitis in Africa; dust in dry season could play a role in facilitating the entry of bacterial pathogens into the host $[10,13]$. We additionally observed a dramatic decrease in the incidence of BI associated with CNSI through time. The overall decreasing trend in BI may be due to the effectiveness of Hib vaccine as part of the current EPI schedule [9], and may additionally be influenced by the usage of vaccines against pneumococcus and meningococcus in the private sector. This is consistent with the downward trend of bacterial CNSI in the countries where Hib immunization has been implemented [27]. The incidence of viral encephalitis has been reported as being higher in summer-fall (rainy) months in the North of Vietnam [16], while a higher incidence of non-BI in our data was identified in the winter and spring (dry) months. This disparity may be associated with the differing climatic conditions between the North and the South of Vietnam. The overall increasing trend in the incidence of non-BI associated CNSI is likely influence by the outbreak of non-BI towards the end of the study period (2015).

Our study contains limitations. CNSI are heterogeneous with respect to the causative agent, mode of transmission, disease presentation, and the corresponding treatment. Each CNSI associated pathogen or group of pathogens is likely to have differences in their spatiotemporal distribution and association with climatic factors, other population-based covariates and thus is likely to have differences in corresponding epidemiological models. Whereas, the ICD10 codes for CNSI from the hospital electronic databases may not accurately reflect specific details of the diagnoses; 
Table 3. Results of the full generalized additive mixed models (GAMM) for paediatric central nervous system (CNS) infections in Ho Chi Minh from 2008 to 2015

\begin{tabular}{|c|c|c|c|c|c|c|c|c|}
\hline & \multicolumn{4}{|c|}{ Presumed bacterial CNS infection } & \multicolumn{4}{|c|}{ Presumed non-bacterial CNS infection } \\
\hline & $\mathrm{RR}$ & LL & UL & $P$ value & $\mathrm{RR}$ & LL & UL & $P$ value \\
\hline Intercept & $0 \cdot 000$ & $0 \cdot 000$ & $1 \cdot 088$ & $0 \cdot 0559$ & $0 \cdot 000$ & $0 \cdot 000$ & $0 \cdot 000$ & $0 \cdot 0023$ \\
\hline Lag 1 month incidence & $1 \cdot 727$ & $1 \cdot 219$ & $2 \cdot 445$ & $0 \cdot 0030$ & $2 \cdot 828$ & 1.766 & $4 \cdot 529$ & $0 \cdot 0001$ \\
\hline Lag 2 month incidence & $0 \cdot 765$ & $0 \cdot 523$ & $1 \cdot 118$ & $0 \cdot 1705$ & $1 \cdot 254$ & $0 \cdot 731$ & $2 \cdot 152$ & $0 \cdot 4134$ \\
\hline Lag 3 month incidence & $1 \cdot 102$ & 0.749 & $1 \cdot 622$ & $0 \cdot 6233$ & $0 \cdot 658$ & $0 \cdot 389$ & $1 \cdot 113$ & $0 \cdot 1234$ \\
\hline Lag 4 month incidence & $0 \cdot 552$ & $0 \cdot 388$ & $0 \cdot 784$ & $0 \cdot 0015$ & $0 \cdot 560$ & $0 \cdot 345$ & $0 \cdot 908$ & $0 \cdot 0218$ \\
\hline Temperature $\left({ }^{\circ} \mathrm{C}\right)$ & $1 \cdot 146$ & 0.996 & $1 \cdot 319$ & $0 \cdot 0621$ & $1 \cdot 100$ & $0 \cdot 900$ & $1 \cdot 344$ & $0 \cdot 3545$ \\
\hline Lag 1 month temperature & $1 \cdot 201$ & $1 \cdot 052$ & $1 \cdot 370$ & $0 \cdot 0084$ & $1 \cdot 506$ & $1 \cdot 243$ & $1 \cdot 824$ & $0 \cdot 0001$ \\
\hline Lag 2 month temperature & $1 \cdot 042$ & 0.909 & $1 \cdot 194$ & $0 \cdot 5571$ & $1 \cdot 000$ & $0 \cdot 823$ & $1 \cdot 213$ & $0 \cdot 9961$ \\
\hline Lag 3 month temperature & $0 \cdot 896$ & $0 \cdot 796$ & $1 \cdot 009$ & $0 \cdot 0752$ & $0 \cdot 864$ & $0 \cdot 739$ & $1 \cdot 010$ & $0 \cdot 0710$ \\
\hline Lag 4 month temperature & $1 \cdot 065$ & $0 \cdot 955$ & $1 \cdot 189$ & $0 \cdot 2629$ & $1 \cdot 199$ & $1 \cdot 039$ & $1 \cdot 385$ & $0 \cdot 0157$ \\
\hline Humidity $(\%)$ & 0.996 & $0 \cdot 962$ & $1 \cdot 032$ & $0 \cdot 8392$ & $0 \cdot 999$ & $0 \cdot 949$ & $1 \cdot 051$ & 0.9596 \\
\hline Lag 1 month humidity & $1 \cdot 020$ & 0.983 & $1 \cdot 057$ & $0 \cdot 2953$ & $1 \cdot 041$ & $0 \cdot 989$ & $1 \cdot 094$ & $0 \cdot 1267$ \\
\hline Lag 2 month humidity & $1 \cdot 001$ & $0 \cdot 966$ & $1 \cdot 038$ & $0 \cdot 9462$ & $1 \cdot 041$ & $0 \cdot 989$ & $1 \cdot 095$ & $0 \cdot 1319$ \\
\hline Lag 3 month humidity & 0.996 & 0.959 & $1 \cdot 033$ & $0 \cdot 8145$ & $1 \cdot 014$ & $0 \cdot 963$ & $1 \cdot 067$ & $0 \cdot 5973$ \\
\hline Lag 4 month humidity & $1 \cdot 014$ & 0.978 & $1 \cdot 052$ & $0 \cdot 4446$ & $1 \cdot 049$ & $0 \cdot 998$ & $1 \cdot 102$ & $0 \cdot 0647$ \\
\hline Rainfall (mm) & $1 \cdot 036$ & $1 \cdot 008$ & $1 \cdot 064$ & $0 \cdot 0137$ & $0 \cdot 992$ & $0 \cdot 957$ & $1 \cdot 029$ & 0.6717 \\
\hline Lag 1 month rainfall & $0 \cdot 999$ & 0.972 & $1 \cdot 025$ & $0 \cdot 9144$ & $1 \cdot 050$ & $1 \cdot 015$ & $1 \cdot 085$ & $0 \cdot 0058$ \\
\hline Lag 2 month rainfall & $1 \cdot 001$ & 0.976 & $1 \cdot 027$ & $0 \cdot 9202$ & $0 \cdot 980$ & $0 \cdot 947$ & $1 \cdot 014$ & $0 \cdot 2485$ \\
\hline Lag 3 month rainfall & $0 \cdot 991$ & 0.968 & $1 \cdot 016$ & $0 \cdot 4868$ & $0 \cdot 960$ & $0 \cdot 930$ & $0 \cdot 990$ & $0 \cdot 0111$ \\
\hline Lag 4 month rainfall & $1 \cdot 019$ & $0 \cdot 991$ & $1 \cdot 048$ & $0 \cdot 1950$ & $1 \cdot 014$ & $0 \cdot 975$ & $1 \cdot 054$ & $0 \cdot 4906$ \\
\hline Water level $(\mathrm{cm})$ & $1 \cdot 011$ & $1 \cdot 001$ & $1 \cdot 020$ & $0 \cdot 0266$ & $1 \cdot 013$ & $1 \cdot 000$ & $1 \cdot 026$ & $0 \cdot 0459$ \\
\hline Lag 1 month water level & $0 \cdot 988$ & 0.977 & $0 \cdot 999$ & $0 \cdot 0364$ & $0 \cdot 996$ & $0 \cdot 981$ & $1 \cdot 011$ & $0 \cdot 5631$ \\
\hline Lag 2 month water level & $1 \cdot 017$ & $1 \cdot 003$ & $1 \cdot 030$ & $0 \cdot 0169$ & $0 \cdot 996$ & $0 \cdot 978$ & $1 \cdot 014$ & $0 \cdot 6310$ \\
\hline Lag 3 month water level & $0 \cdot 998$ & 0.985 & $1 \cdot 011$ & $0 \cdot 8009$ & $1 \cdot 017$ & $1 \cdot 000$ & $1 \cdot 034$ & $0 \cdot 0614$ \\
\hline Lag 4 month water level & $1 \cdot 005$ & 0.993 & $1 \cdot 016$ & $0 \cdot 4292$ & $1 \cdot 016$ & $1 \cdot 000$ & $1 \cdot 032$ & $0 \cdot 0541$ \\
\hline Time trend (month) & $0 \cdot 613$ & $0 \cdot 384$ & 0.978 & $0 \cdot 0441$ & $2 \cdot 043$ & $1 \cdot 194$ & $3 \cdot 495$ & $0 \cdot 0113$ \\
\hline
\end{tabular}

Results of fixed effects are shown in the table.

$P$ values for seasonality for presumed bacterial and non-bacterial CNS infection $=0 \cdot 1990$ and $0 \cdot 5268$, respectively.

Adjusted $R^{2}$ for presumed bacterial and non-bacterial CNS infection $=0.78$ and 0.74 , respectively.

RR: (incidence) rate ratio for one unit increase of covariates. LL and UL: lower and upper limits of $95 \%$ confidence interval.

there were very limited data regarding specific pathogens and as a result, stratifying hospital data with 'microbiological sub-classes' or pathogens is not possible. Acknowledging this limitation, we stratified our analyses by two large groups of particular clinical relevance (BI and non-BI) since the main effort for CNSI diagnosis clinically is to differentiate between bacterial and non-bacterial etiologies and thus the ICD10 codes might better reflect this. We were additionally unable to identify specific spatial risk factors for paediatric CNSI. This may be due to the fact that BI and non-BI groups are generalized and the information on various characteristics of the districts was limited. Lastly, we were unable to obtain the data on vaccine deployment by district per year to provide a detailed description of the spatiotemporal effect of immunization on the incidence of paediatric CNSI. Therefore, more detailed prospective studies examining the different population factors associated with information regarding specific CNSI pathogens, such as JE and pneumococcal meningitis, and the interplay with their spatiotemporal risk factors are recommended. Furthermore, surveillance systems for specific CNSI pathogens as well as CNSI-related vaccine deployment should be improved in this setting.

In conclusion, we found that BIs are the most common form of CNSI in newborns and infants aged $<1$ year in HCMC. We additionally found that the urban districts surrounding the referral hospitals and two districts on the outskirts of the city had the highest incidences of paediatric CNSI. Notably, the incidence of CNSI was higher in the dry season and significantly associated with temperature, rainfall and water level of the rivers within the city. Our 

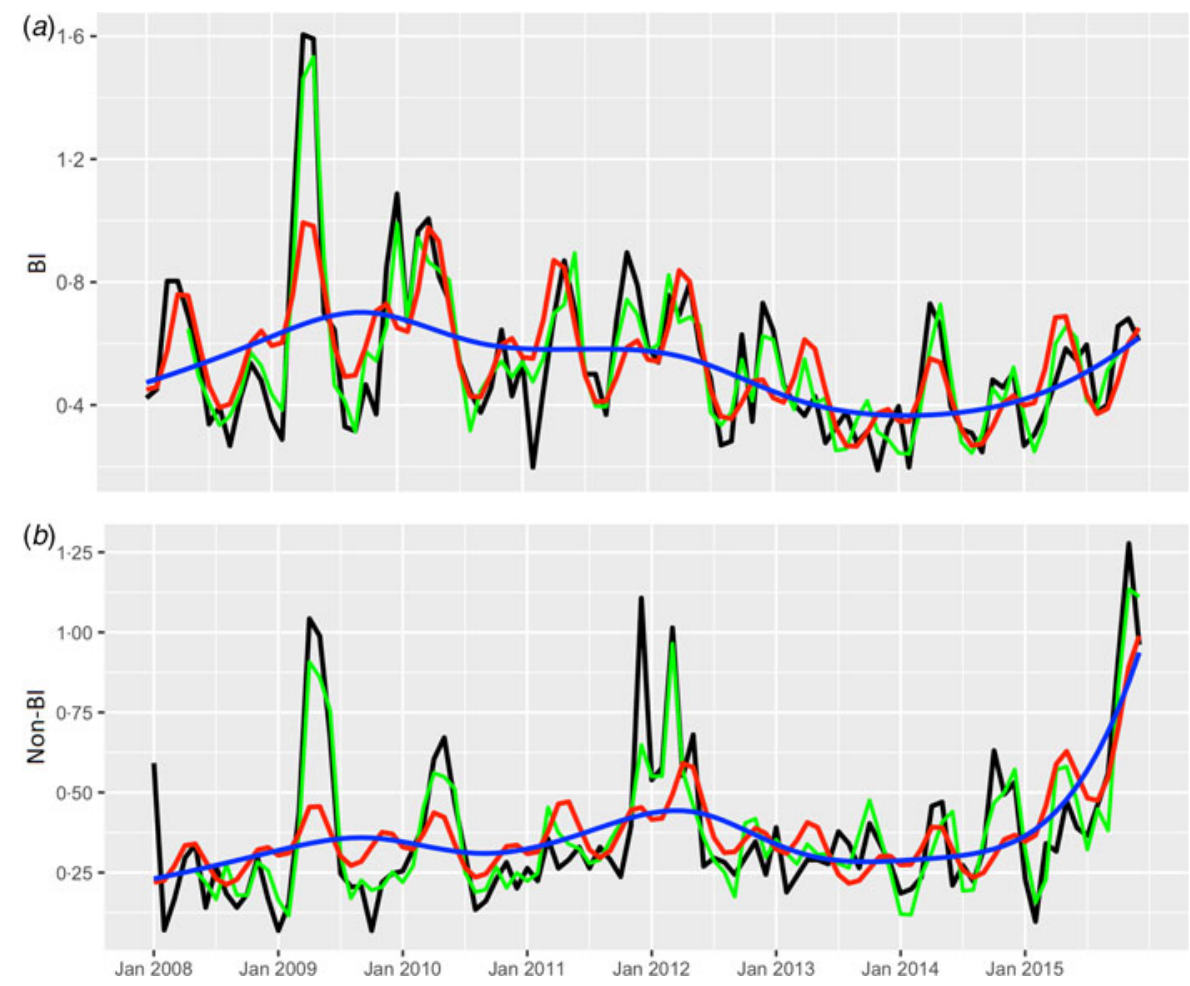

Fig. 3. The monthly incidence time series of paediatric presumed bacterial and non-bacterial CNSIs. (a) Plot showing the smoothed seasonal cycle (red line), the citywide monthly incidence (number of monthly cases per 100000 total population of that month) time trend (blue line) of the presumed bacterial CNSI (BI) from the basic Poisson generalized additive mixed model (GAMM) and the predicted monthly BI incidence (green line) from the full Poisson GAMM with observed monthly BI incidence (black line). (b) Plot showing the smoothed seasonal cycle (red line), the citywide monthly incidence (number of monthly cases per 100000 total population of that month) time trend (blue line) of the presumed non-bacterial CNSI (non-BI) from the basic GAMM and the predicted monthly non-BI incidence (green line) from the full Poisson GAMM with observed monthly non-BI incidence (black line).

findings suggest that further prospective studies and better surveillance are needed in this setting to add further guidance into where and when the resources for the prevention, control, and treatment of paediatric CNSI should be directed. This is particularly important, as the vaccines against the bacterial and viral pathogens of CNSI are costly and not routinely available in this location.

\section{SUPPLEMENTARY MATERIAL}

The supplementary material for this article can be found at https://doi.org/10.1017/S095026881700228X

\section{ACKNOWLEDGEMENTS}

The authors are grateful to the staff of Children's Hospital 1, Children's Hospital 2 and The Hospital for Tropical Diseases for providing the databases used in this study. This work was funded by the Wellcome Trust. SB is a Sir Henry Dale Fellow, jointly funded by the Wellcome Trust and the Royal Society $(100087 / \mathrm{Z} / 12 / \mathrm{Z})$. NTH is currently funded by a Mervyn Susser Fellowship at Columbia University Medical Center, USA. The funders had no role in study design, data collection, data analysis, data interpretation, writing of the report, or decision to submit the article for publication.

\section{AUTHORS' CONTRIBUTIONS}

This study was conceptualized by NTH, HMTV, LNTN, NTD and SB. Data were provided by LNTN, HMTV, NTD, TMP, VMQ, NNQM, TAT, NTH, HMT, NVVC. NTH, AT and DK analyzed the data. NTH, KTT, DH and SB wrote the manuscript. All authors read and approved the final version of the manuscript.

\section{DECLARATION OF INTEREST}

None. 


\section{ETHICS STATEMENT}

Ethical approval was granted from all of the three study hospitals: Children Hospital 1, Children Hospital 2 and the Hospital for Tropical Diseases. The authors assert that all procedures contributing to this work comply with the ethical standards of the relevant national and institutional committees on human experimentation and with the Helsinki Declaration of 1975, as revised in 2008.

\section{REFERENCES}

1. Chong HT, Tan CT. Epidemiology of central nervous system infections in Asia, recent trends. Neurology Asia 2005; 10:7-11.

2. Newton CR. Burden of central nervous system infections. In: Singhi P, Griffin DE, eds. Central Nervous System Infections in Childhood. London, UK: Mac Keith Press, 2014, pp. 1-7.

3. Wang $\mathbf{H}$, et al. Global, regional, and national life expectancy, all-cause mortality, and cause-specific mortality for 249 causes of death, 1980-2015: a systematic analysis for the Global Burden of Disease Study 2015. The Lancet 2016; 388(10053): 1459-1544.

4. Ho Dang Trung N, et al. Aetiologies of central nervous system infection in Viet Nam: a prospective provincial hospital-based descriptive surveillance study. PLoS ONE 2012; 7(5): e37825.

5. van Tan L, et al. Viral etiology of encephalitis in children in Southern Vietnam: results of a one-year prospective descriptive study. PLoS Neglected Tropical Diseases 2010; 4(10): e854.

6. Anh DD, et al. Haemophilus influenzae type b meningitis among children in Hanoi, Vietnam: epidemiologic patterns and estimates of $\mathrm{H}$. influenzae type $\mathrm{b}$ disease burden. The American Journal of Tropical Medicine and Hygiene 2006; 74(3): 509-515.

7. Jit M, et al. Thirty years of vaccination in Vietnam: impact and cost-effectiveness of the national expanded programme on immunization. Vaccine 2015; 33(Suppl 1): A233-A239.

8. WHO. Data, Statistics and Graphics. WHO. 2016 (cited 17 Jan 2017); Available from: http://www.who.int/ immunization/monitoring_surveillance/data/en/.

9. Le P, et al. Cost-effectiveness of Haemophilus influenzae type b vaccine in Vietnam. Vaccine 2015; 33(36): 4639-4646.

10. Agier L, et al. Seasonality of meningitis in Africa and climate forcing: aerosols stand out. Journal of the Royal Society, Interface 2013; 10(79): 20120814.

11. García-Pando CP, et al. Soil dust aerosols and wind as predictors of seasonal meningitis incidence in Niger. Environmental Health Perspectives 2014; 122(7): 679-686.

12. Paireau $\mathbf{J}$, et al. Seasonal dynamics of bacterial meningitis: a time-series analysis. The Lancet Global Health 2016; 4(6): e370-e377.

13. Paireau J, et al. Spatio-temporal factors associated with meningococcal meningitis annual incidence at the health centre level in Niger, 2004-2010. PLoS Neglected Tropical Diseases 2014; 8(5): e2899.

14. Zhao X, et al. Japanese encephalitis risk and contextual risk factors in southwest China: a Bayesian hierarchical spatial and spatiotemporal analysis. International Journal of Environmental Research and Public Health 2014; 11(4): 4201-4217.

15. Li X, et al. The Spatio-temporal distribution of Japanese encephalitis cases in different age groups in Mainland China, 2004-2014. PLoS Neglected Tropical Diseases 2016; 10(4): e0004611.

16. Lee HS, et al. Seasonality of viral encephalitis and associated environmental risk factors in Son La and Thai Binh Provinces in Vietnam from 2004 to 2013. The American Journal of Tropical Medicine and Hygiene 2017; 96(1):110-117.

17. Erlanger TE, et al. Past, present, and future of Japanese encephalitis. Emerging Infectious Diseases 2009; 15(1): 1-7.

18. Statistical Office in Ho Chi Minh City. Statistical Yearbook of Ho Chi Minh City 2015. Ho Chi Minh City: Ho Chi Minh City Statistical Office, 2015.

19. Loc PD. Ministry of Natural Resources and Environment of the Socialist Republic of Vietnam. Ha Noi, 2013. Available from: www.monre.gov.vn/.

20. Jarvis A, et al. Hole-filled Seamless SRTM Data V4. International Centre for Tropcial Agriculture (CIAT). 2008. Available from: http://srtm.csi.cgiar.org/.

21. Bivand R, Hauke J, Kossowski T. Computing the Jacobian in Gaussian spatial autoregressive models: an illustrated comparison of available methods. Geographical Analysis 2013; 45(2): 150-179.

22. Kulldorff M, Nagarwalla N. Spatial disease clusters: detection and inference. Statistics in Medicine 1995; 14 (8): 799-810.

23. Cameron AC, Trivedi PK. Microeconometrics Using Stata, vol. 5. Stata Press books, 2009, pp. 706. Available from: http://stata.biz/news/statanews.23.4.pdf.

24. Lin X, Zhang D. Inference in generalized additive mixed models by using smoothing splines. Journal of the Royal Statistical Society: Series B 1999; 61(2): 381-400.

25. Wood SN. Generalized Additive Models: An Introduction with $R$, 2nd edn. Florida: Chapman \& Hall/CRC Texts in Statistical Science, 2017, pp. 476.

26. R Core Team. R: A Language and Environment for Statistical Computing [Internet]. Vienna, Austria: R Foundation for Statistical Computing, 2016. Available from: https://www.r-project.org/.

27. Dash N, et al. Epidemiology of meningitis in Al-Ain, United Arab Emirates, 2000-2005. International Journal of Infectious Diseases 2007; 11(4): 309-312.

28. Abucejo-Ladesma E, et al. Serious community-acquired paediatric infections in rural Asia (Bohol Island, Philippines): bacterial meningitis in children less than 5 years of age. Scandinavian Journal of Infectious Diseases 2007; 39(11-12): 983-989.

29. Caserta MT. Neonatal Bacterial Meningitis [Internet]. Pediatrics - Merck Manuals Professional Edition, 2015 (cited 24 Sep 2016). Available from: http://www. merckmanuals.com/professional/pediatrics/infections-inneonates/neonatal-bacterial-meningitis. 